\title{
Association between physical function and long-term care in community-dwelling older and oldest people: the SONIC study
}

Werayuth Srithumsuk', Mai Kabayama', Kayo Godai', Nonglak Klinpudtan', Ken Sugimoto², Hiroshi Akasaka², Yoichi Takami², Yasushi Takeya², Koichi Yamamoto², Saori Yasumoto ${ }^{3}$, Yasuyuki Gondo ${ }^{3}$, Yasumichi Arai ${ }^{4}$, Yukie Masui ${ }^{5}$, Tatsuro Ishizaki ${ }^{5}$, Hiroshi Shimokata ${ }^{6}$, Hiromi Rakugi ${ }^{2}$ and Kei Kamide ${ }^{1,2^{*}}$

\begin{abstract}
Background: Preventing the need for long-term care (LTC) by identifying physical function risk factors are important to decrease the LTC burden. The objective of this study was to investigate whether grip strength and/or walking speed, which are components of the frailty definition, are associated with LTC in community-dwelling older and oldest people.

Methods: The participants were 1098 community-dwelling older and oldest people who had not received LTC at the baseline. The endpoint was receiving LTC after the baseline survey. The independent variables were grip strength and walking speed, and participants were divided into two groups based on these variables. The confounding factors were age, sex, the Japanese version of the Montreal Cognitive Assessment (MoCA-J), hypertension, diabetes mellitus, stroke, joint diseases, living alone, body mass index, and serum albumin. We calculated the hazard ratio of receiving LTC using the Cox proportional hazard model.

Results: Among the 1098 participants, 107 (9.7\%) newly received LTC during the follow-up. Regarding the physical function, only slow walking speed was significantly correlated with LTC after adjusting for all confounding factors except the MoCA-J score $(H R=1.74,95 \% \mathrm{Cl}=1.10-2.75, P=.018)$. However, slow walking speed was still a risk factor for $L T C$ after adjusting for the MoCA-J score and other confounding factors $(H R=1.64,95 \% \mathrm{Cl}=1.03-2.60, P=.037)$.

Conclusions: The findings from this study may contribute to a better understanding of slow walking speed as a factor related to LTC, which might be a criterion for disability prevention and could serve as an outcome measure for physical function in older people.
\end{abstract}

Keywords: Frailty, Grip strength, Long-term care, Older people, Walking speed

\footnotetext{
* Correspondence: kamide@sahs.med.osaka-u.ac.jp

'Department of Health Promotion System Sciences, Division of Health Sciences, Graduate School of Medicine, Osaka University, Osaka, Japan

${ }^{2}$ Department of Geriatric and General Medicine, Graduate School of Medicine, Osaka University, Osaka, Japan

Full list of author information is available at the end of the article
}

(C) The Author(s). 2020 Open Access This article is licensed under a Creative Commons Attribution 4.0 International License, which permits use, sharing, adaptation, distribution and reproduction in any medium or format, as long as you give appropriate credit to the original author(s) and the source, provide a link to the Creative Commons licence, and indicate if changes were made. The images or other third party material in this article are included in the article's Creative Commons licence, unless indicated otherwise in a credit line to the material. If material is not included in the article's Creative Commons licence and your intended use is not permitted by statutory regulation or exceeds the permitted use, you will need to obtain permission directly from the copyright holder. To view a copy of this licence, visit http://creativecommons.org/licenses/by/4.0/ The Creative Commons Public Domain Dedication waiver (http://creativecommons.org/publicdomain/zero/1.0/) applies to the data made available in this article, unless otherwise stated in a credit line to the data. 


\section{Background}

In 2000, Japan developed a system of long-term care (LTC) that rendered LTC a universal entitlement for every Japanese citizen aged 65 or older [1]. As a rapid increase in the users of LTC is leading to financial constraints, the Japanese government revised the LTC system in 2006 to concentrate on prevention strategies for those with lower-level needs and those at risk of requiring $\mathrm{LTC}$ in the future [2], to minimize these problems. However, previous studies showed that the following are predictive factors for certification to receive LTC: advanced age, impaired walking ability, deterioration in instrumental activities of daily living (IADL), cognitive impairment, treatment of underlying diseases, number of medications, history of falls, loss of excretory ability, functional inactivity, low social activity, poor self-reported health, not able to work, and depression [3-5]. Among such factors, progressive deterioration in physical function and activities of daily living are major health concerns of older people [6]. Therefore, screening for an impaired physical function is very important in the early identification of health risks among older people.

Fried et al. [7] proposed the phenotype model, which measures frailty using the components of the Cardiovascular Health Study (CHS) to describe the age-related decline in physical capacities based on the following criteria: (1) unintentional weight loss, (2) self-reported exhaustion, (3) low grip strength, (4) low physical activity, and (5) slow walking speed. According to this model, the presence of three or more indicates frailty with regard to Japan; Satake et al. [8] advocated a Japanese version of CHS (J-CHS) criteria, which can also identify frailty. The J-CHS's component and criteria are similar to the original phenotype model. Previous studies of frailty and adverse outcomes examined falls, hospitalizations, disability, death, and admission to LTC facilities $[7,9]$. However, no such study has tracked communitydwelling older people developing a need for LTC under home care or community-based care. In a study by Lee et al. [10], it was demonstrated that the combined use of grip strength and walking speed was accurate, precise, specific, and more sensitive for the Fried frailty phenotype than other possible combinations and suggested that these measures could be applied in a primary care setting. More significantly, grip strength and walking speed measurements do not require special equipment, they are not time-consuming or intrusive, and they are helpful in making decisions about interventions and care allocation [11].

To our knowledge, no studies have determined the association of physical function with the incidence of the certified need of care in the national LTC system using handgrip strength and walking speed measurements in older and oldest Japanese people living in the community because in previous studies, data were collected based on self-reports $[3,4]$ and the follow-up period was between 3 and 4 years [3-5]. Therefore, to prevent older people from showing a declining function in the future, provide them with efficient and effective care, and delay its clinical consequences, investigating the relationship between physical function using grip strength and walking speed as indicators of the need for LTC is useful for community-dwelling older people. Moreover, longitudinal studies can provide information on changes in physical function in older people, such as transitions, trajectories, and factors associated with LTC. Consequently, the objective of this study was to investigate whether grip strength and/or walking speed, which are components of the frailty definition, are associated as physical function indicators with LTC in community-dwelling older and oldest people.

\section{Methods \\ Participants}

The study participants were recruited from the Septuagenarians, Octogenarians, Nonagenarians Investigation with Centenarians (SONIC) study, a longitudinal analysis involving the collection of data in the community at the baseline and follow-up every 3 years of a prospective cohort study ongoing since 2010 [12].

The inclusion criteria of this study were as follows: (1) they were free of LTC needs at the baseline, (2) having LTC data at the baseline and follow-up, (3) no missing data for each potential confounding factor, (4) without a history of Parkinson's disease, and (5) free of the need for walking aids at the baseline. All data were collected at the baseline (2010-2012), while LTC data were collected at the baseline and during follow-up (2013-2018). About $69.63 \%$ of the participants were followed up every 3 years (wave 1 -wave 3 ), meaning that participants aged 70 years were followed for 8 years while those aged 80 and 90 years were followed for 7 and 6 years, respectively. The follow-up for the remainder varied from 3 to 8 years. At the baseline, a total of 2245 participants in all age groups $(69-71$ years old $=1000,79-81$ years old $=$ 973, and 89-91 years old $=272$ ) were included, but only 1098 participants met the inclusion criteria (Fig. 1).

\section{The LTC assessment}

Full details regarding the LTC system in Japan were described previously [1]. The care need levels for LTC services are categorized into the following 7 levels: support need levels 1 and 2 and care need levels 1-5. The higher the level of care need, the more severe the conditions, requiring higher levels of care and services to avoid functional limitations and promote independent living. We identified participants who were receiving some level of LTC services as having received LTC. 


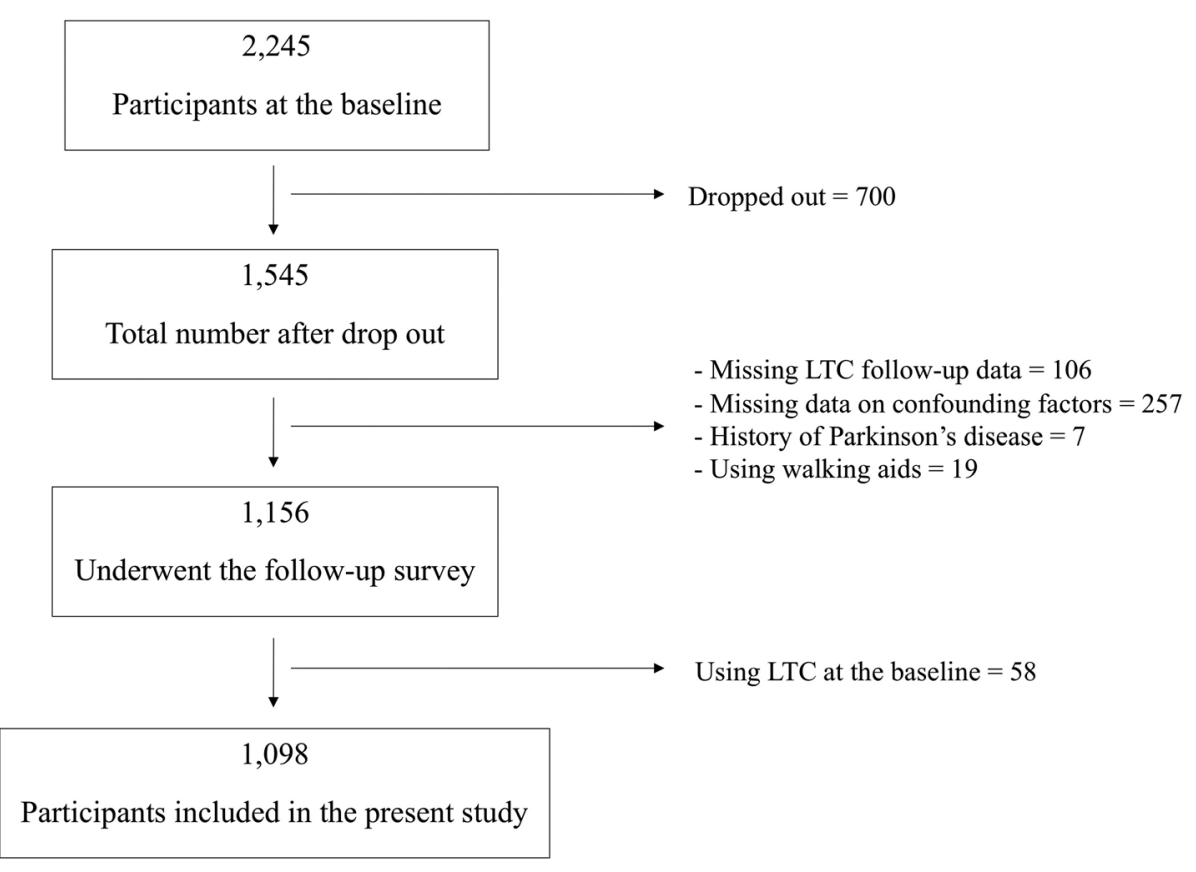

Fig. 1 Participants included in the study

\section{Grip Strength}

The participants' grip strength was performed by trained physical therapists. The dominant grip strength was measured using the Smedley hand dynamometer (Model YD-100; Yagami Ltd., Tokyo, Japan). Measurements were taken in a sitting posture twice, with arms resting against the body. In the analysis, the average of the first and second measurements was used. Weak grip strength was defined as $<26 \mathrm{~kg}$ for males and $<18 \mathrm{~kg}$ for females [8].

\section{Walking speed}

The participants' walking speed was implemented by trained physical therapists. The time taken to walk along a 2.44-m course was measured on a walking speed scale. Another measurement was obtained using a digital timer, and the two measurements were combined to give the average walking speed. Slow walking speed was defined according to a cutoff $<1.0 \mathrm{~m} / \mathrm{s}$ [8].

\section{Potential confounding factors}

Potential confounding factors taken into consideration in the study included age and sex. Living alone was noted in the two groups (living alone and living together with others). A blood sample was obtained, and biochemical tests in grams/deciliter units determined serum albumin levels.

History of stroke and joint diseases was classified in accordance with their yes/no responses. Body mass index (BMI) was measured and formulated as the weight in kilograms divided by the square of the height in meters $\left(\mathrm{kg} / \mathrm{m}^{2}\right)$.

Hypertension (HT) was diagnosed in accordance with the 2019 guideline of the Japanese Society of Hypertension [13], characterized by systolic blood pressure (BP) $\geq$ $140 \mathrm{mmHg}$ and diastolic BP $\geq 90 \mathrm{mmHg}$ or antihypertensive drug use.

Diabetes mellitus (DM) was defined as fasting blood glucose $\geq 126 \mathrm{mg} / \mathrm{dL}$, casual blood glucose $\geq 200 \mathrm{mg} / \mathrm{dL}$, hemoglobin A1c $\geq 6.5 \%$, or antidiabetic drug use by the Japan Diabetes Society [14].

The cognitive function assessment of the participants' was performed by trained psychologists using the Japanese version of the Montreal Cognitive Assessment (MoCA-J) [15]. The total scores of MoCA-J (0-30 points) were used for assessment of the cognitive function. A higher score indicated greater cognitive function.

\section{Statistical analysis}

The outcome event was the need for LTC. Cases were censored during the follow-up period. First, we carried out a descriptive analysis of the samples; Pearson's chisquare or Fisher's exact test for categorical variables and the independent $t$ test for continuous variables were used to compare baseline characteristics between groups who had received and not received LTC.

Cox proportional hazard regression analyses were performed using the following 3 models to estimate hazard ratios (HRs) and 95\% confidence intervals (95\% CI). Model 1 was constructed using the grip strength and 
walking speed as independent variables. Model 2 included age, sex, HT, DM, stroke, joint diseases, living alone, BMI, and serum albumin as independent variables in addition to grip strength and walking speed. Model 3 included the MoCA-J score in addition to model 2, which was called the adjusted model. Moreover, cox proportional hazard regression analyses were also implemented following stratification by age. $P$ values of $<0.05$ were considered significant. These statistical analyses were carried out with SPSS Statistics 26.0 (IBM Japan, Tokyo, Japan).

\section{Results}

In the baseline survey, 2245 participants were recruited, but only 1098 (48.91\%) had not received LTC at the baseline and met the inclusion criteria, as shown in Fig. 1. After a follow-up of the LTC status, 107 (9.7\%) participants had used LTC services. When the participants were grouped based on receiving LTC after follow-up, the independent physical function was also significant based on a weak grip strength on comparing those who received and those who did not receive LTC (53.3 vs. $35.7 \%$, respectively; $P<.001$ ). Similarly, the slow walking speed in those who received LTC was also assigned a higher percentage than in those not receiving LTC (75.7 vs. $58.2 \%$, respectively; $P<.001$ ) (Table 1).

Table 2 shows findings from the Cox proportional hazard regression analyses. The analysis of independent physical function variables showed that only slow walking speed was significantly correlated with LTC after adjusting for all confounding factors except the MoCA-J score ( $\mathrm{HR}=1.74,95 \%$ $\mathrm{CI}=1.10-2.75, P=.018)$. However, slow walking speed was still a risk factor for LTC after adjusting for the MoCA-J score and other confounding factors $(\mathrm{HR}=1.64$, $95 \% \mathrm{CI}=1.03-2.60, P=.037)$. Moreover, other variables affecting the need for LTC included a lower MoCA-J score $(\mathrm{HR}=0.93,95 \% \mathrm{CI}=0.88-0.98, P=.005)$, an advanced age (80 years old, $\mathrm{HR}=4.56,95 \% \mathrm{CI}=2.55-8.16, P<.001 ; 90$ years old, $\mathrm{HR}=8.84,95 \% \mathrm{CI}=3.81-20.52, P<.001$; with 70 years old as a reference), and a female sex, with females showing a higher risk than males $(\mathrm{HR}=1.63,95 \% \mathrm{CI}=$ $1.07-2.49, P=.023)$. In addition, on comparing cumulative hazards between the groups, both grip strength and walking speed are shown in Figs. 2 and 3. Moreover, adjusted Cox proportional hazard regression analyses following age stratification (only 70 and 80 years old) were implemented (Additional file 1: Table S1 \& Additional file 2: Table S2), but the analysis of those in their 90s is not shown because no one in the normal walking speed group received LTC and the sample size was too small.

\section{Discussion}

The principal finding of the present study was that those receiving LTC among Japanese community-dwelling older and oldest people showed a significantly slower walking speed as an independent physical function variable. Additional predictors of receiving LTC were a lower MoCA-J score, an advanced age, and a female sex.

Long-term care was correlated with only a slow walking speed but was not significant for a weak grip strength, and this finding agreed with that of Chen et al. [6] who investigated the use of walking speed and grip strength to predict the cause of mortality and found that walking speed function impairment was a more powerful predictor of all-cause mortality at 3 years than grip strength. The study by Makizako et al. [16] also demonstrated a slower walking speed, expected following activity limitation even in independent older adults with no IADL limitation. This indicates that walking speed is worth considering as a predictive marker of IADL limitation. Walking speed was, therefore, superior to other physical performance tests, such as grip strength, in identifying populations at a higher risk of all-cause mortality. At the same time, the combination of walking speed with grip strength had no better prognostic benefit than using walking speed only in predicting mortality from all causes [6].

Walking speed is, according to Shinkai [17], a strong predictor of IADL impairment in older people. This indicates that retaining the walking capacity while living independently is important for older adults. Owing to the decrease in walking speed and IADL, older people often continue to lose out on opportunities to take part in recreational activities and other social life aspects. For older people, whether they need some support or are completely independent, maintaining that level of independence requires strategies such as maintaining the functional ADL and remaining socially active through regular leisure activities that promote maintenance of the walking capacity [18]. Moreover, a slow walking speed was recognized as a better predictor of cumulative deficits over multiple physiological systems, which may also be more useful in identifying older adults with higher health risk profiles and the cause of the need for LTC. From this point of view, a slow walking speed has had a significant effect on the need for LTC certification compared with weak grip strength, as walking speed is a complex activity requiring sophisticated coordination of multiple body systems [19] as well as adequate coordination to maintain strength production, structural support, movement, and balance control [20].

The need for LTC is related to not only independent factors such as a slow walking speed but also other variables such as a lower MoCA-J score, an advanced age, and a female sex. In the study of Nishiguchi et al. [21], they found that cognitive decline is an independent predictor of new LTC requirement certifications, and the severity of the cognitive decline in the elderly is positively associated with 
Table 1 Comparison of baseline characteristics between subjects in non-received LTC and those received LTC groups during followup $(n=1,098)$

\begin{tabular}{|c|c|c|c|c|}
\hline Characteristics & $\begin{array}{l}\text { Total } \\
\boldsymbol{N}(\%) \\
\end{array}$ & Non-received LTC $(\boldsymbol{n}=991 ; 90.3 \%)$ & Received LTC $(\boldsymbol{n}=107 ; 9.7 \%)$ & $\boldsymbol{P}$ value \\
\hline \multicolumn{5}{|l|}{ Grip strength $^{a}$} \\
\hline Normal & $687(62.6)$ & $637(64.3)$ & $50(46.7)$ & $<.001^{\mathrm{d}}$ \\
\hline Male & $389(56.6)$ & $367(57.6)$ & $22(44.0)$ & \multirow[t]{2}{*}{$.075^{d}$} \\
\hline Female & $298(43.4)$ & $270(42.4)$ & $28(56.0)$ & \\
\hline Weak & $411(37.4)$ & $354(35.7)$ & $57(53.3)$ & \\
\hline Male & $152(37.0)$ & $135(38.1)$ & $17(29.8)$ & \multirow[t]{2}{*}{$.241^{d}$} \\
\hline Female & $259(63.0)$ & $219(61.9)$ & $40(70.2)$ & \\
\hline \multicolumn{5}{|l|}{ Walking speed $^{\mathrm{b}}$} \\
\hline Normal & $440(40.1)$ & $414(41.8)$ & $26(24.3)$ & \multirow[t]{2}{*}{$<.001^{\mathrm{d}}$} \\
\hline Slow & $658(59.9)$ & $577(58.2)$ & $81(75.7)$ & \\
\hline MoCA-J score (Mean \pm SD) & $23.00 \pm 3.44$ & $23.17 \pm 3.35$ & $21.44 \pm 3.84$ & $<.001$ \\
\hline \multicolumn{5}{|l|}{ Age } \\
\hline $70 \mathrm{y} / \mathrm{o}$ & $523(47.6)$ & $508(51.3)$ & $15(14.0)$ & \multirow[t]{3}{*}{$<.001^{\mathrm{C}}$} \\
\hline $80 \mathrm{y} / \mathrm{o}$ & $531(48.4)$ & $452(45.6)$ & 79 (73.8) & \\
\hline $90 \mathrm{y} / \mathrm{o}$ & $44(4.0)$ & $31(3.1)$ & $13(12.1)$ & \\
\hline \multicolumn{5}{|l|}{ Sex } \\
\hline Male & $541(49.3)$ & $502(50.7)$ & 39 (36.4) & \multirow[t]{2}{*}{$.006^{\mathrm{d}}$} \\
\hline Female & $557(50.7)$ & $489(49.3)$ & $68(63.6)$ & \\
\hline \multicolumn{5}{|l|}{ HT } \\
\hline No & $299(27.2)$ & $278(28.1)$ & $21(19.6)$ & \multirow[t]{2}{*}{$.068^{\mathrm{d}}$} \\
\hline Yes & $799(72.8)$ & $713(71.9)$ & $86(80.4)$ & \\
\hline \multicolumn{5}{|l|}{ DM } \\
\hline No & $931(84.8)$ & $843(85.1)$ & $88(82.2)$ & \multirow[t]{2}{*}{$.440^{c}$} \\
\hline Yes & $167(15.2)$ & $148(14.9)$ & $19(17.8)$ & \\
\hline \multicolumn{5}{|l|}{ Stroke } \\
\hline No & $1043(95.0)$ & $943(95.2)$ & $100(93.5)$ & \multirow[t]{2}{*}{$.444^{c}$} \\
\hline Yes & $55(5.0)$ & $48(4.8)$ & $7(6.5)$ & \\
\hline \multicolumn{5}{|l|}{ Joint diseases } \\
\hline No & $824(75.0)$ & $756(76.3)$ & $68(63.6)$ & \multirow[t]{2}{*}{$.006^{\mathrm{d}}$} \\
\hline Yes & $274(25.0)$ & $235(23.7)$ & $39(36.4)$ & \\
\hline \multicolumn{5}{|l|}{ Living alone } \\
\hline No & $909(82.8)$ & $832(84.0)$ & $77(72.0)$ & \multirow[t]{2}{*}{$.002^{c}$} \\
\hline Yes & $189(17.2)$ & $159(16.0)$ & $30(28.0)$ & \\
\hline BMI (Mean \pm SD) & $22.73 \pm 2.89$ & $22.75 \pm 2.88$ & $22.50 \pm 3.00$ & .386 \\
\hline Serum albumin (Mean \pm SD) & $4.37 \pm 0.27$ & $4.38 \pm 0.27$ & $4.30 \pm 0.27$ & .005 \\
\hline
\end{tabular}

Independent $t$ test for continuous variable

$B M I$ body mass index, DM diabetes mellitus, HT hypertension, LTC long-term care, MoCA-J The Japanese version of the Montreal Cognitive Assessment, SD standard deviation, $y / o$ years old

aWeakness was defined as a maximum grip strength $<26 \mathrm{~kg}$ in males and $<18 \mathrm{~kg}$ in females

${ }^{b}$ Slowness was defined as a gait speed $<1.0 \mathrm{~m} / \mathrm{s}$.

${ }^{c} P$ values from Pearson's chi-square test

${ }^{\mathrm{d}} P$ values from Fisher's exact test

LTC requirement certification in Japan. In an LTC setting, cognitive functioning may be a robust predictor, because dysfunction can impair one's ability to complete ADLs, as well as one's ability to engage in safety precautions (e.g., preventing falls). Thus, changes in cognitive functioning may possibly further impair the physical function [22]. 
Table 2 Cox proportional hazard regression analyses of receiving LTC $(n=1098)$

\begin{tabular}{|c|c|c|c|c|c|c|}
\hline \multirow[t]{2}{*}{ Variables } & \multicolumn{2}{|c|}{ Model $1^{a}$} & \multicolumn{2}{|l|}{ Model $2^{b}$} & \multicolumn{2}{|c|}{ Model $3^{c}$} \\
\hline & $H R$ & $95 \% \mathrm{Cl}$ & $\mathrm{HR}$ & $95 \% \mathrm{Cl}$ & $H R$ & $95 \% \mathrm{Cl}$ \\
\hline $\begin{array}{l}\text { Weak grip strength } \\
\text { ref. normal }\end{array}$ & $2.12^{* * *}$ & $1.45-3.09$ & 1.34 & $0.90-2.01$ & 1.23 & $0.82-1.85$ \\
\hline $\begin{array}{l}\text { Slow walking speed } \\
\text { ref. normal }\end{array}$ & $2.24^{* * *}$ & $1.44-3.49$ & $1.74^{*}$ & $1.10-2.75$ & $1.64^{*}$ & $1.03-2.60$ \\
\hline MoCA-J score & - & - & - & - & $0.93^{* *}$ & $0.88-0.98$ \\
\hline \multicolumn{7}{|l|}{ Age $($ ref. $=70 \mathrm{y} / \mathrm{o})$} \\
\hline $80 \mathrm{y} / \mathrm{o}$ & - & - & $5.11^{* * *}$ & $2.87-9.11$ & $4.56^{* * *}$ & $2.55-8.16$ \\
\hline $90 \mathrm{y} / \mathrm{o}$ & - & - & $11.35^{* * *}$ & $4.98-25.85$ & $8.84^{* * *}$ & $3.81-20.52$ \\
\hline Sex (ref. = male) & - & - & $1.63^{*}$ & $1.07-2.49$ & $1.63^{*}$ & $1.07-2.49$ \\
\hline HT & - & - & 1.01 & $0.61-1.68$ & 0.99 & $0.60-1.64$ \\
\hline DM & - & - & 1.12 & $0.67-1.88$ & 1.13 & $0.68-1.90$ \\
\hline Stroke & - & - & 1.41 & $0.64-3.08$ & 1.44 & $0.66-3.15$ \\
\hline Joint diseases & - & - & 1.11 & $0.74-1.67$ & 1.18 & $0.79-1.78$ \\
\hline Living alone & - & - & 1.34 & $0.86-2.10$ & 1.42 & $0.91-2.23$ \\
\hline BMI & - & - & 1.01 & $0.94-1.08$ & 1.00 & $0.93-1.08$ \\
\hline Serum albumin & - & - & 0.65 & $0.31-1.39$ & 0.72 & $0.35-1.50$ \\
\hline
\end{tabular}

$B M I$ body mass index, $C l$ confidence interval, DM diabetes mellitus, HR hazard ratio, HT hypertension, LTC long-term care, MoCA-J The Japanese version of the Montreal Cognitive Assessment, ref. reference, $y / o$ years old

${ }^{*} P<.05$

${ }^{* *} P<.01$

${ }^{* * *} P<.001$

${ }^{a}$ Model 1 was unadjusted for physical function (weak grip strength and slow walking speed)

${ }^{b}$ Model 2 was adjusted for physical function by age, sex, HT, DM, stroke, joint diseases, living alone, BMI, and serum albumin

${ }^{\mathrm{C}}$ Model 3 was adjusted for physical function by MoCA-J score, age, sex, HT, DM, stroke, joint diseases, living alone, BMI, and serum albumin

Advanced age- and sex-related differences in walking speed also emerged. In the study of Wu et al. [23], determinants of the need for LTC among older Taiwanese adults were examined, and they found that advanced age was a predictive factor for receiving LTC. This basically illustrates that people who are older usually show a decrease in health, and this, in effect, leads to the increased use of health care like LTC services [24]. While sex-related slow walking speed in females was a higher risk of the need for LTC compared with males. This phenomenon can be explained by women showing impaired exercise performance in the domains of lower extremity strength, balance, and ambulation, which means that a sedentary lifestyle will cause low social activity and then ADL or IADL limitations; subsequently, LTC needs among older people can increase [25].

In the present study, several limitations have to be mentioned. First, the participants in the study are not a

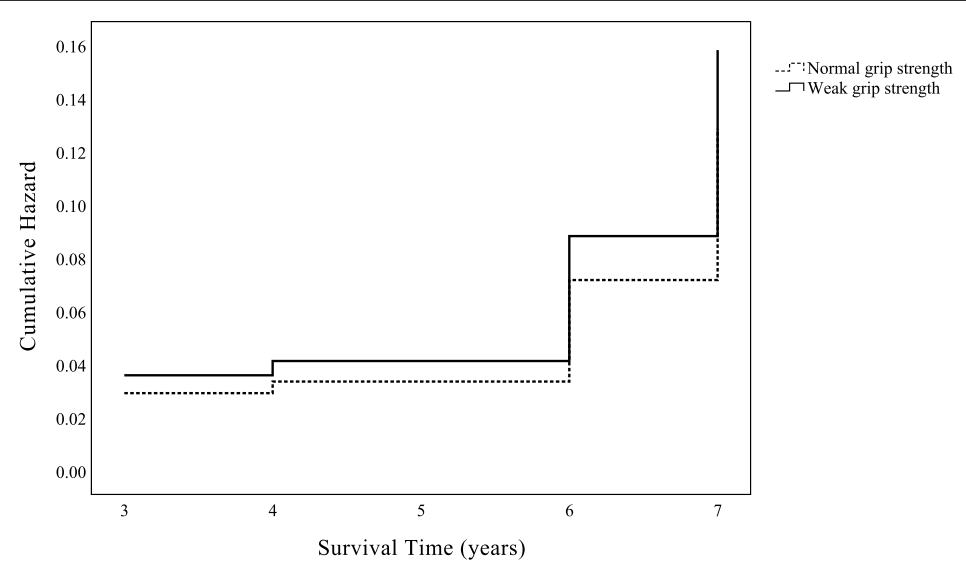

Fig. 2 Cumulative hazard of grip strength on long-term care 


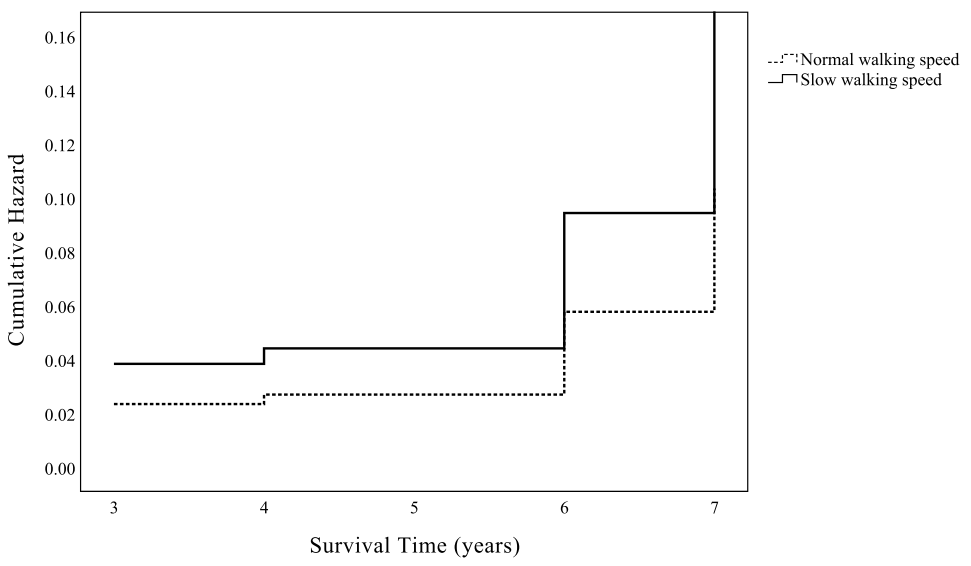

Fig. 3 Cumulative hazard of walking speed on long-term care

representative sample of the general population of older Japanese people but rather were volunteers and, thus, possibly more health conscious. As such, selection bias might be inevitable in the present study. Second, our study population was limited, including only non-institutionalized, community-dwelling, age-homogeneous Japanese citizens in restricted areas, most of whom were mentally healthy and without dementia despite the sample being taken from a basic residential registry. Lastly, the number of people with a need for LTC may have been underestimated because this was measured using LTC insurance in the present study. People with such a need may thus have been grouped as not requiring LTC. Moreover, LTC data were collected by participants' self-report. Information from national LTC database system may have more accuracy.

\section{Conclusion}

The findings of this study may contribute to a better understanding of factors related to LTC. In particular, walking speed could be a useful indicator to classify older people at high risk of requiring LTC, which may be a criterion for disability prevention interventions and could serve as an outcome measure for physical function studies in older people.

\section{Supplementary information}

Supplementary information accompanies this paper at https://doi.org/10. 1186/s12199-020-00884-3.

Additional file 1: Table S1. Cox proportional hazard regression analyses of those receiving LTC $(n=523)$ (70 years old).

Additional file 2: Table S2. Cox proportional hazard regression analyses of those receiving LTC $(n=531)$ (80 years old).

\section{Abbreviations}

ADL: Activities of daily living; BMI: Body mass index; BP: Blood pressure; CHS: Cardiovascular Health Study; Cl: Confidence interval; DM: Diabetes mellitus; HR: Hazard ratio; HT: Hypertension; IADL: Instrumental activities of daily living; J-CHS: The Japanese version of Cardiovascular Health Study;
LTC: Long-term care; MoCA-J: The Japanese version of the Montreal Cognitive Assessment; SONIC: The Septuagenarians, Octogenarians, Nonagenarians Investigation with Centenarians study

\section{Acknowledgements}

We are grateful to all SONIC participants who participated in these studies. We sincerely appreciate all staff involved in the SONIC study, especially Yumiko Aoshima and Tae Matsue for their secretarial work and support. The members of the SONIC Study Group (in addition to the authors) are as follows: Toshiaki Sekiguchi, Kentaro Tanaka, Tomoko Noma, Kazuya Taira, Naoko Wada, and Atsuko Higuchi at the Division of Health Sciences, Osaka University Graduate School of Medicine; Serina Yokoyama, Satomi Maeda, Motonori Nagasawa, and Taku Fujimoto at the Department of Geriatric and General Medicine, Osaka University Graduate School of Medicine; Kennichi Matsuda, Taiji Ogawa, Yuichi Mihara, Masahiro Kitamura, and Yoshinobu Maeda at Osaka University Graduate School of Dentistry.

\section{Authors' contributions}

Srithumsuk, Kabayama, Gondo, Godai, Rakugi, and Kamide: concept, designed, and developed the overall research plan. All authors carried out the data collection. Srithumsuk, Kabayama, Godai, Klinpudtan, and Kamide conducted the analysis and interpretation of data. Sritumsuk, Kabayama, Godai, and Kamide wrote and had responsibility for the final contents of the manuscript. All authors read and approved the final manuscript.

\section{Funding}

This work was supported by grants-in-aid from the Ministry of Education, Culture, Sports, Science, and Technology of Japan (K.K.: 19 K07888, M.K.: 19 K11138) and from the Ministry of Health, Labour and Welfare (H.S.).

Availability of data and materials

The datasets used and/or analyzed during the current study are available from the corresponding author on reasonable request.

\section{Ethics approval and consent to participate}

The SONIC research was approved by the Osaka University Graduate School of Medicine, Dentistry, and Human Sciences (Osaka, Japan) and the Tokyo Metropolitan Geriatric Hospital and Institute of Gerontology (Tokyo, Japan) Institutional Review Board. Before beginning the survey, informed consent was obtained from all study participants on-site. Anonymity was ensured.

Consent for publication

Not applicable.

Competing interests

The authors declare that they have no competing interests. 


\section{Author details}

${ }^{1}$ Department of Health Promotion System Sciences, Division of Health Sciences, Graduate School of Medicine, Osaka University, Osaka, Japan. ${ }^{2}$ Department of Geriatric and General Medicine, Graduate School of Medicine, Osaka University, Osaka, Japan. ${ }^{3}$ Department of Clinical Thanatology and Geriatric Behavioral Science, Graduate School of Human Sciences, Osaka University, Osaka, Japan. ${ }^{4}$ Center for Supercentenarian Medical Research, Keio University School of Medicine, Tokyo, Japan. ${ }^{5}$ Tokyo Metropolitan Geriatric Hospital and Institute of Gerontology, Tokyo, Japan. ${ }^{6}$ Graduate School of Nutritional Sciences, Nagoya University of Arts and Sciences, Aichi, Japan.

Received: 1 July 2020 Accepted: 17 August 2020

Published online: 01 September 2020

\section{References}

1. Tsutsui T, Muramatsu N. Care-needs certification in the long-term care insurance system of Japan. J Am Geriatr Soc. 2005;53:522-7.

2. Tsuji T, Kondo K, Kondo N, Aida J, Takagi D. Development of a risk assessment scale predicting incident functional disability among older people: Japan Gerontological Evaluation Study. Geriatr Gerontol Int. 2018;18: 1433-8

3. Fujiwara Y, Amano H, Kumagai S, et al. Physical and psychological predictors for the onset of certification of long-term care insurance among older adults living independently in a community a 40-month follow-up study. [Nippon kōshū eisei zasshi]. Japanese J Public Heal. 2006;53:77-91.

4. Hirai H, Kondo K, Ojima T, Murata C. Examination of risk factors for onset of certification of long-term care insurance in community-dwelling older people: AGES project 3-year follow-up study. [Nippon kōshū eisei zasshi]. Japanese J public Heal. 2009;56:501-12.

5. Ohmori-Matsuda K, Hozawa A, Sone T, et al. Depression and the risk of long-term care insurance certification: the Tsurugaya project. [Nippon kōshū eisei zasshi]. Japanese J public Heal. 2010;57:538-49.

6. Chen PJ, Lin MH, Peng LN, et al. Predicting cause-specific mortality of older men living in the veterans home by handgrip strength and walking speed: a 3-year, prospective cohort study in Taiwan. J Am Med Dir Assoc. 2012;13: $517-21$

7. Fried LP, Tangen CM, Walston J, et al. Frailty in older adults: evidence for a phenotype. J Gerontol A-Biol. 2001;56:M146-57.

8. Satake S, Shimada $\mathrm{H}$, Yamada M, et al. Prevalence of frailty among community-dwellers and outpatients in Japan as defined by the Japanese version of the Cardiovascular Health Study criteria. Geriatr Gerontol Int. 2017;17:2629-34.

9. Clegg A, Young J, Iliffe S, Rikkert MO, Rockwood K. Frailty in elderly people. Lancet. 2013;381:752-62.

10. Lee $L$, Patel $T$, Costa $A$, et al. Screening for frailty in primary care: accuracy of gait speed and hand-grip strength. Can Fam Physician. 2017;63:e51-7.

11. Hoogendijk EO, Afilalo J, Ensrud KE, Kowal P, Onder G, Fried LP. Frailty: implications for clinical practice and public health. Lancet. 2019;394:1365-75.

12. Gondo Y, Masui Y, Kamide K, Ikebe K, Arai Y, Ishizaki T. SONIC study: A longitudinal cohort study of the older people as part of a centenarian study. In: Pachana NA, editor. . Encyclopedia of Geropsychology, Springer Science+Business Media Singapore: Singapore; 2016.

13. Saruta T. The Japanese Society of Hypertension guidelines for the management of hypertension (JSH 2004). Nippon Rinsho Japanese J Clin Med. 2005;63:952-8.

14. Haneda M, Noda M, Origasa H, et al. Japanese Clinical Practice Guideline for Diabetes 2016. J Diabetes Investig. 2018:9:657-97.

15. Fujiwara $Y$, Suzuki $H$, Yasunaga $M$, et al. Brief screening tool for mild cognitive impairment in older Japanese: validation of the Japanese version of the Montreal Cognitive Assessment. Geriatr Gerontol Int. 2010;10:225-32.

16. Makizako $\mathrm{H}$, Shimada $\mathrm{H}$, Tsutsumimoto $\mathrm{K}$, et al. Social frailty in communitydwelling older adults as a risk factor for disability. J Am Med Dir Assoc. 2015;16:1003.e7-1003.e11.

17. Shinkai S. Walking speed as a good predictor for the onset of functional dependence in a Japanese rural community population. Age Ageing. 2000; 29:441-6.

18. Fukuma M, Shiwaku K. A prospective study of frailty, mortality and required level of care in elderly requiring support. Shimane J Med Sci. 2014;30:59-68.
19. Rosso AL, Sanders JL, Arnold AM, et al. Multisystem physiologic impairments and changes in gait speed of older adults. J Gerontol Ser A Biol Sci Med Sci. 2015;70:319-24

20. Studenski S, Perera S, Patel K, et al. Gait speed and survival in older adults. J Am Med Assoc. 2011;305:50-8.

21. Nishiguchi S, Yamada M, Sonoda T, et al. Cognitive decline predicts longterm care insurance requirement certification in community-dwelling older Japanese adults: a prospective cohort study. Dement Geriatr Cogn Disord Extra. 2013;3:312-9.

22. Kane $\mathrm{KD}$, Yochim $\mathrm{BP}$, Lichtenberg PA. Depressive symptoms and cognitive impairment predict all-cause mortality in long-term care residents. Psychol Aging. 2010;25:446-52.

23. Wu CY, Hu HY, Huang N, Fang YT, Chou YJ, Li CP. Determinants of longterm care services among the elderly: a population-based study in Taiwan. PLoS One. 2014:9:e89213.

24. Meijer CD, Wouterse B, Polder J, Koopmanschap M. The effect of population aging on health expenditure growth: a critical review. Eur J Ageing. 2013;10: 353-61.

25. Gardner AW, Montgomery PS. Differences in exercise performance and leisure-time physical activity in older caucasians and african-americans. Clin Med Geriatr. 2008;19:1-7.

\section{Publisher's Note}

Springer Nature remains neutral with regard to jurisdictional claims in published maps and institutional affiliations.
Ready to submit your research? Choose BMC and benefit from:

- fast, convenient online submission

- thorough peer review by experienced researchers in your field

- rapid publication on acceptance

- support for research data, including large and complex data types

- gold Open Access which fosters wider collaboration and increased citations

- maximum visibility for your research: over $100 \mathrm{M}$ website views per year

At $\mathrm{BMC}$, research is always in progress.

Learn more biomedcentral.com/submissions 